\title{
Keck Array and BICEP3: Spectral Characterization of $5000+$ Detectors
}

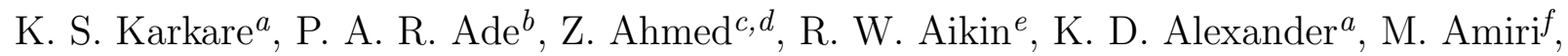
D. Barkats ${ }^{g}$, S. J. Benton ${ }^{h}$, C. A. Bischoff ${ }^{a}$, J. J. Bock ${ }^{e, i}$, J. A. Bonetti ${ }^{i}$, J. A. Brevik ${ }^{e}$, I. Buder ${ }^{a}$, E. W. Bullock ${ }^{j}$, B. Burger ${ }^{f}$,J. Connors ${ }^{a}$, B. P. Crill ${ }^{e, i}$, G. Davis ${ }^{f}$, C. D. Dowell ${ }^{i}$, L. Duband ${ }^{k}$, J. P. Filippini ${ }^{e}$, S. T. Fliescher ${ }^{l}$, S. R. Golwala ${ }^{e}$, M. S. Gordon ${ }^{a}$, J. A. Grayson ${ }^{c}$,

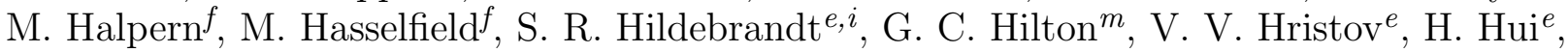
K. D. Irwin ${ }^{c, d, m}$, J. H. Kang ${ }^{c}$, E. Karpel ${ }^{c}$, S. Kefelie, S. A. Kernasovskiy ${ }^{c}$, J. M. Kovac $^{a}$, C. L. Kuo ${ }^{c, d}$, E. M. Leitch ${ }^{n}$, M. Lueker ${ }^{e}$, P. Mason ${ }^{e}$, K. G. Megerian ${ }^{i}$, C. B. Netterfield ${ }^{h, o}$, H. T. Nguyen ${ }^{i}$, R. O’Brient ${ }^{i}$, R. W. Ogburn IV ${ }^{c, d}$, C. Pryke ${ }^{l, j}$, C. D. Reintsema ${ }^{m}$, S. Richter $^{a}$, R. Schwarz ${ }^{l}$, C. D. Sheehy ${ }^{l, n}$, Z. K. Staniszewski ${ }^{e, i}$, R. V. Sudiwala ${ }^{b}$, G. P. Teply ${ }^{e}$, K. L. Thompson ${ }^{c, d}$, J. E. Tolan ${ }^{c}$, A. D. Turner ${ }^{i}$, A. G. Vieregg ${ }^{a, n}$, A. Weber ${ }^{i, e}$, C. L. Wong ${ }^{a}$, W. L. K. $\mathrm{Wu}^{c}$, and K. W. Yoon ${ }^{c, d}$

${ }^{a}$ Harvard-Smithsonian Center for Astrophysics, Cambridge, Massachusetts 02138, USA

${ }^{b}$ School of Physics and Astronomy, Cardiff University, Cardiff, CF24 3AA, United Kingdom

${ }^{c}$ Department of Physics, Stanford University, Stanford, California 94305, USA

${ }^{d}$ Kavli Institute for Particle Astrophysics and Cosmology, SLAC National Accelerator

Laboratory, Menlo Park, California 94025, USA

${ }^{e}$ Department of Physics, California Institute of Technology, Pasadena, California 91125, USA

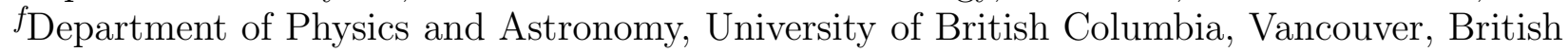

Columbia, V6T 1Z1, Canada

${ }^{g}$ Joint ALMA Observatory, Vitacura, Santiago, Chile

${ }^{h}$ Department of Physics, University of Toronto, Toronto, Ontario, M5S 1A7, Canada

${ }^{i}$ Jet Propulsion Laboratory, Pasadena, California 91109, USA

${ }^{j}$ Minnesota Institute for Astrophysics, University of Minnesota, Minneapolis, Minnesota 55455, USA

${ }^{k}$ Service des Basses Températures, Commisariat à l'Energie Atomique, 38054 Grenoble, France

${ }^{l}$ Department of Physics, University of Minnesota, Minneapolis, Minnesota 55455, USA

${ }^{m}$ National Institute of Standards and Technology, Boulder, Colorado 80305, USA

${ }^{n}$ University of Chicago, Chicago, Illinois 60637, USA

${ }^{\circ}$ Canadian Institute for Advanced Research, Toronto, Ontario, M5G 1Z8, Canada

\begin{abstract}
The inflationary paradigm of the early universe predicts a stochastic background of gravitational waves which would generate a $B$-mode polarization pattern in the cosmic microwave background (CMB) at degree angular scales. Precise measurement of $B$-modes is one of the most compelling observational goals in modern cosmology. Since 2011, the Keck Array has deployed over 2500 transition edge sensor (TES) bolometer detectors at 100 and $150 \mathrm{GHz}$ to the South Pole in pursuit of degree-scale B-modes, and BICEP3 will follow in 2015 with 2500 more at $100 \mathrm{GHz}$. Characterizing the spectral response of these detectors is important for controlling systematic effects that could lead to leakage from the temperature to polarization signal, and for understanding potential
\end{abstract}

Send correspondence to K. S. Karkare: 60 Garden St., MS 42, Cambridge, MA 02138, USA. E-mail: kkarkare@cfa.harvard.edu

Millimeter, Submillimeter, and Far-Infrared Detectors and Instrumentation for Astronomy VII, edited by Wayne S. Holland, Jonas Zmuidzinas, Proc. of SPIE Vol. 9153, 91533B - (c) 2014 SPIE $\cdot$ CCC code: $0277-786$ X/14/\$18 $\cdot$ doi: $10.1117 / 12.2056779$ 
coupling to atmospheric and astrophysical emission lines. We present complete spectral characterization of the Keck Array detectors, made with a Martin-Puplett Fourier Transform Spectrometer at the South Pole, and preliminary spectra of BICEP3 detectors taken in lab. We show band centers and effective bandwidths for both Keck Array bands, and use models of the atmosphere at the South Pole to cross check our absolute calibration. Our procedure for obtaining interferograms in the field with automated 4-axis coupling to the focal plane represents an important step towards efficient and complete spectral characterization of next-generation instruments more than 10000 detectors.

Keywords: Inflation, Gravitational waves, Cosmic microwave background, Polarization, BICEP, Keck Array

\section{INTRODUCTION}

A brief period of accelerated exponential expansion in the early Universe, while a radical extrapolation from understood physics, naturally solves the flatness, horizon, and monopole problems of standard cosmology. This inflationary period also explains the origin of structure by stretching quantum fluctuations to macroscopic scales. Inflation predicts perturbations to the metric in both scalars (density waves) and tensors (gravitational waves). Both types of perturbations will affect the polarization of the cosmic microwave background (CMB) at last scattering: scalars can only generate a gradient-type ( $E$-mode) pattern, while tensors can also generate a curltype ( $B$-mode) pattern. Measurement of $B$-mode power in the CMB at degree angular scales would be direct evidence for an inflationary period, and the amplitude of the signal - parametrized by $r$, the tensor-to-scalar ratio - would indicate the energy scale of inflation.

The BICEP2 experiment has recently reported a detection of $B$-mode polarization at degree angular scales from three years of observation at $150 \mathrm{GHz}{ }^{1}$ Assuming a simple inflationary gravitational wave model this corresponds to $r=0.20_{-0.05}^{+0.07}$, while accounting for the contribution of foreground contamination will shift this value downward by an amount which will be better constrained with upcoming datasets. Confirming this signal and determining its spectral characteristics are therefore extremely urgent observational goals. The Keck Array and BICEP3 are the next experiments in the BICEP/Keck series, all of which employ a similar, targeted experimental design. The Keck Array ${ }^{2,3}$ has been operating with a full complement of five BICEP2-type receivers since 2012. In 2012 and 2013, all receivers operated at $150 \mathrm{GHz}$. In December 2013, two of the receivers were converted to $100 \mathrm{GHz}$. BICEP3, featuring the same total number of detectors as the Keck Array in a single cryostat at $100 \mathrm{GHz}$, will deploy to South Pole this year and begin observing in 2015. ${ }^{4}$

In these proceedings, we describe the spectral response of the Keck Array and BICEP3. In the 2013-2014 deployment season, we obtained spectra of all Keck Array detectors in situ at the South Pole with a fielddeployable Fourier Transform Spectrometer (FTS). We have obtained preliminary spectra of BICEP3 in lab. Understanding the spectral response of the detectors is necessary for characterizing systematic effects which could potentially cause a spurious polarization signal: relative gain mismatch between co-located detectors which can leak the much stronger CMB temperature signal into polarization, and out-of-band coupling to atmospheric or astrophysical emission lines. When characterizing the frequency dependence of a sky signal, knowledge of detector bandpasses determines the expected amplitudes of different astrophysical components such as CMB, dust, or synchrotron emission. Both the $100 \mathrm{GHz}$ and $150 \mathrm{GHz}$ detector bandpasses have been designed to avoid atmospheric molecular lines. Even with this precaution, weak response to a bright molecular line could present another systematic to the CMB measurement. Atmospheric lines such as oxygen's at $118 \mathrm{GHz}$ contribute excess optical loading, leading to reduced sensitivity; astrophysical lines such as carbon monoxide's at $115 \mathrm{GHz}$ could produce a signal which is correlated with the molecular component of the interstellar medium. ${ }^{5}$ Detailed beam characterization $^{6-8}$ and careful mitigation in analysis ${ }^{9}$ are necessary for reducing systematic effects to levels small compared to an observed $B$-mode signal.

In Section 2 we review the band-defining elements of the Keck Array receivers. In Section 3 we describe the apparatus used to obtain detector spectra, and in Section 4 we present the results of our measurements. Finally, in Section 5 we use our measured spectra in conjunction with simulated South Pole skies from the am modeling software* to simulate realistic South Pole skies and cross check our absolute calibration routines.

\footnotetext{
*https://www.cfa.harvard.edu/ spaine/am/
} 


\section{SPECTRAL RESPONSE}

The choice of frequency bands is a tradeoff between sensitivity, atmospheric and foreground information, physical placement of detectors on the focal plane, and optical performance. In this section, we briefly review the elements of the BICEP2 and Keck Array receivers which determine the spectral response: the physical antenna pattern, the band-defining filter, and the edge filter. See the BICEP2 instrument paper ${ }^{10}$ and detectors paper ${ }^{11}$ for more details about this design, which is common to BICEP2, Keck Array, BICEP3, and SPIDER.

\subsection{Antenna Design}

Our focal planes consist of tiles of monolithically fabricated antenna-array-coupled TES (transition edge sensor) bolometers. In each pixel, microwave power couples to a planar phased array antenna composed of slot subradiators carved into a superconducting niobium ground plane. The co-located sub-radiators sample orthogonal polarizations, which are summed coherently in a microstrip feed network to accomplish beam synthesis in lieu of other methods such as horns or lenslets. With no additional filtering, the antenna network design achieves $>30 \%$ fractional bandwidth. ${ }^{12}$

\subsection{Band-Defining Filters}

Before termination on the TES bolometer, each polarization's microstrip feed passes through a band-defining filter. The 3-pole filters consist of lumped inductors which couple to their neighbors via a T-network of capacitors, and are a compromise between maximizing integrated power and limiting dielectric loss from multiple passes through each resonator. We have two design frequency bands: $150 \mathrm{GHz}$ (with $25 \%$ fractional bandwidth, defined at the $3 \mathrm{~dB}$ points), and $95 \mathrm{GHz}$; these have been chosen to avoid the spectral lines of oxygen at $118.8 \mathrm{GHz}$ and water at $183.3 \mathrm{GHz}$ in order to reduce atmospheric loading, and $1 / f$ noise from clouds and other fluctuations in the atmospheric brightness.

\subsection{Edge Filter}

We have added a cold $(4 \mathrm{~K})$ metal mesh low-pass filter ${ }^{13}$ to reduce coupling to submillimeter radiation. In the past we have observed a "blue leak" corresponding to radiation directly coupling to the TES island; this filter removes sensitivity to higher frequencies. For the $150 \mathrm{GHz}$ detectors we use a filter with a cutoff at $8 \mathrm{~cm}^{-1}$, and for $100 \mathrm{GHz}$ the cutoff is at $4 \mathrm{~cm}^{-1}(240$ and $120 \mathrm{GHz}$, respectively).

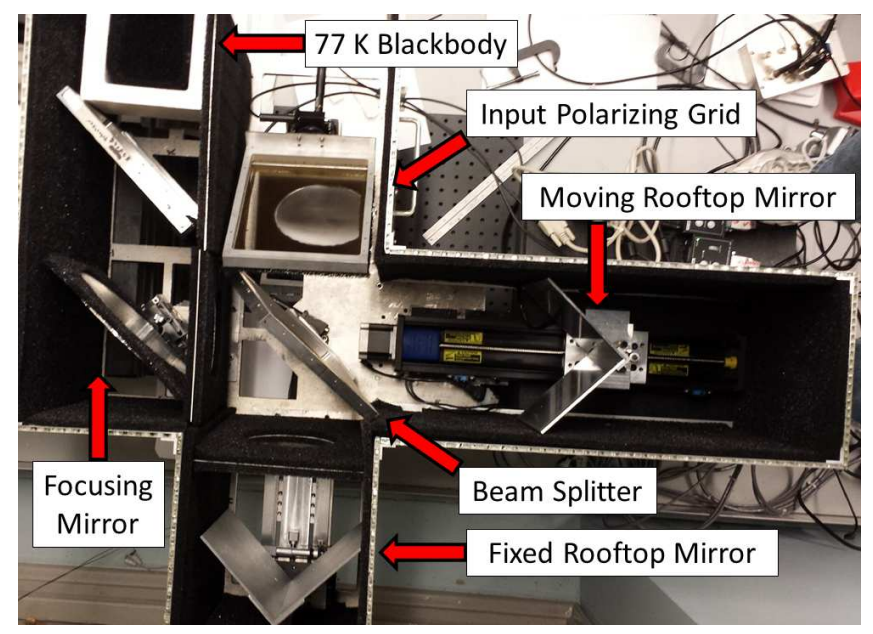

Figure 1. Martin-Puplett Fourier Transform Spectrometer (FTS) in lab. In the time-reverse sense, the detector beam enters the FTS via the input grid, is split into two polarizations, travels different path lengths, is recombined, and terminates on either a room-temperature blackbody or a $77 \mathrm{~K}$ blackbody, depending on the polarization state. The difference between the two sources determines the brightness of the modulated signal. 


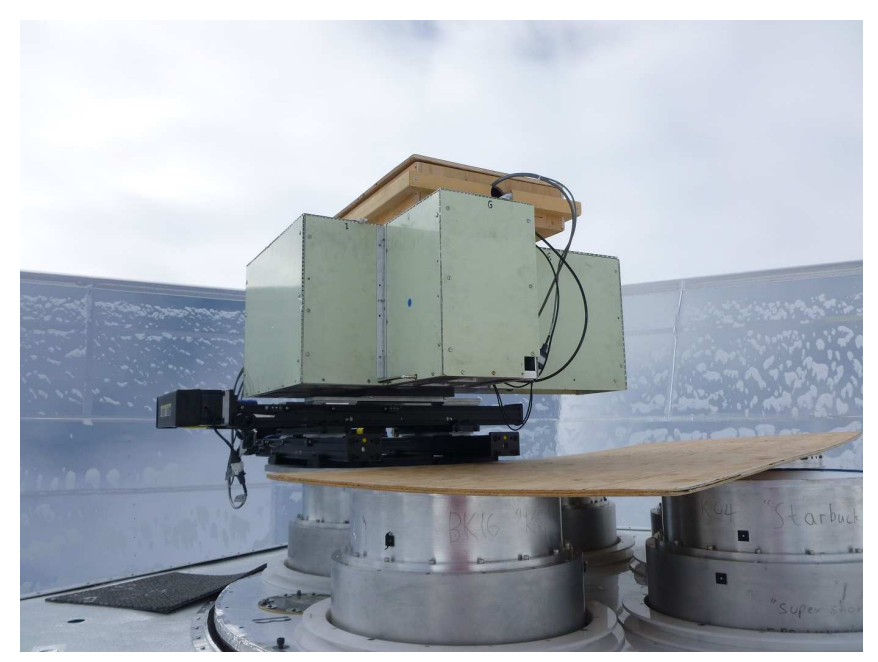

Figure 2. The FTS taking spectra of a single Keck Array receiver on the DASI mount at South Pole. It sits atop a translation stage which allows it to illuminate any detector on the focal plane.

\section{MEASUREMENTS}

\subsection{Fourier Transform Spectrometer}

To take spectra of our detectors, we have constructed a field-deployable Martin-Puplett Fourier Transform Spectrometer. ${ }^{14,15}$ Figure 1 shows the FTS in lab. The detector to be characterized lies beneath the FTS and illuminates the 5" diameter FTS aperture with a collimated beam (in the time reverse sense). This beam intercepts an input polarizing grid which redirects a single (vertical) polarization into the interferometer. Another polarizing grid at $45^{\circ}$ with respect to the input grid splits the beam and redirects each half onto arms of different path lengths. Rooftop mirrors at the ends of the arms reflect the beams back and change the polarization state by $90^{\circ}$ so that the beams are recombined after a second pass through the $45^{\circ}$ grid. The moving mirror is controlled by a Velmex BiSlide translation stage ${ }^{\dagger}$. The recombined beam then illuminates a focusing mirror. The focused light from the mirror is again split into two polarizations; one terminates on Eccosorb HR-10 microwave absorber ${ }^{\ddagger}$ at ambient temperature, and the other on HR-10 held at $77 \mathrm{~K}$ with liquid nitrogen. It is the difference in temperature between the two sources that leads to an interference pattern in the detector when the path length is changed.

\subsection{Coupling to Detectors}

The total spectral power received by a detector with effective area $A_{e}$ and observing a source with frequency dependence $S(\nu)$ is given by $P(\nu)=\frac{1}{2} \int_{4 \pi} A_{e}(\theta, \phi) S(\nu, \theta, \phi) d \Omega$. If the source is a blackbody in the RayleighJeans limit and fills the beam, $S(\nu, \theta, \phi)=B(\nu)=\frac{2 k T}{\lambda^{2}}$. For a single-moded antenna, $A_{e}=\frac{\lambda^{2}}{4 \pi}$. We then have $P(\nu)=k T$, which is independent of frequency. ${ }^{16}$ If the source does not fill the beam, the directional dependence introduces a frequency dependence in the detected power. Due to the limitations on the size of the FTS aperture, it is not possible to fill the beams of the $26 \mathrm{~cm}$ apertures of the Keck Array. However, it is possible to angle the FTS aperture such that it illuminates each detector through the center of its beam.

The FTS is attached to a Velmex BiSlide X-Y translation stage which mounts directly to the vacuum window of a Keck Array receiver. The center of the FTS aperture is thus free to move to any location over the window, intercepting the beam center of any detector on the focal plane. The polarizing aperture grid of the FTS is then angled in both "elevation" and "azimuth" to match the exit angle of the detector beam. The grid is controlled with a Thorlabs goniometer and rotation stage ${ }^{\S}$. This 4-axis coupling both allows each detector on

\footnotetext{
${ }^{\dagger}$ Velmex, Inc., Bloomfield, NY 14469

${ }^{\ddagger}$ Emerson \& Cuming Microwave Products, Randolph, MA 02368

$\S$ Thorlabs Inc., Newton, NJ 07860
} 
the focal plane to be illuminated with high signal-to-noise, and mitigates significant frequency dependence in the resultant spectra. Since the input grid (in the time reverse sense) reflects a single polarization, it can be oriented to illuminate the "A" and "B" (orthogonally polarized) detectors separately, or a combination of the two. When taking spectra of many detectors at once, we choose to orient the FTS at $45^{\circ}$ with respect to the detector polarization axes to illuminate both types of detectors equally.

\subsection{In Situ Measurements of the Keck Array}

Since our FTS is designed to mount to a complete receiver, it can easily be used in lab, on the Keck Array in the DASI mount, or on BICEP3 in the BICEP mount. Calibrations made when the telescope is in its final observing configuration offer the most realistic measurements which are directly applicable to science data. To this end, we have taken spectra of Keck Array receivers while they are in the DASI mount at the South Pole, just before the start of CMB observations. The measurements presented in these proceedings took place during December 2013 and correspond to the 2014 observing season. Figure 2 shows the FTS taking spectra of a single receiver; complete characterization of one receiver takes about 10 hours.

\subsection{In-Lab Measurements of BICEP3}

At the time of these proceedings, BICEP3 has been built and is being tested in a replica of the BICEP mount at Harvard University. ${ }^{4}$ We have taken spectra of 3 detector modules with a total of $\sim 120$ active detectors. We plan to measure BICEP3 spectra in situ at the South Pole in the 2014-2015 deployment season.
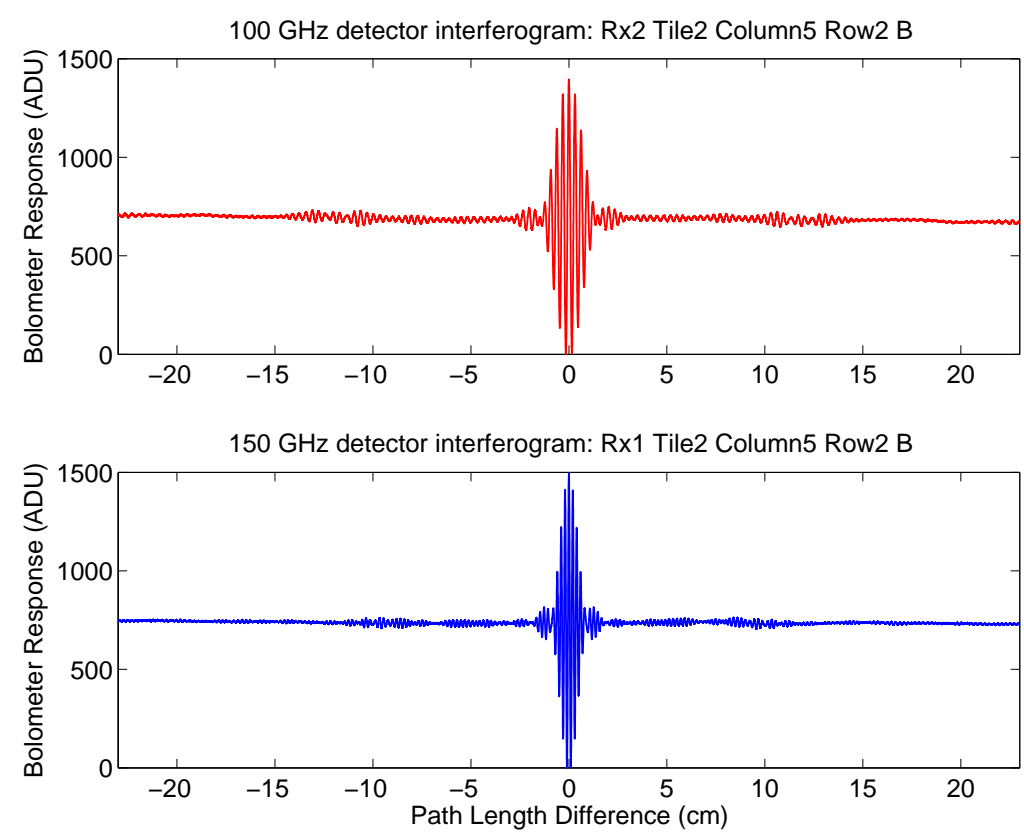

Figure 3. Example raw interferograms of Keck Array detectors taken on the mount, for a $100 \mathrm{GHz}$ receiver (above) and a $150 \mathrm{GHz}$ reciever (below). Noise levels with no signal modulation are several ADU.

\section{RESULTS}

\subsection{Spectra}

We produce detector interferograms (traces of power as a function of mirror position) by recording the raw detector timestreams and encoder readings from the moving mirror encoder. Figure 3 shows example interferograms taken from two Keck Array detectors at 100 and $150 \mathrm{GHz}$ in their observing configurations at South Pole. The amplitudes in these interferograms are typical of the best-illuminated pointings for each detector - we measure the modulated signal for most detectors with an extremely high signal-to-noise ratio. 


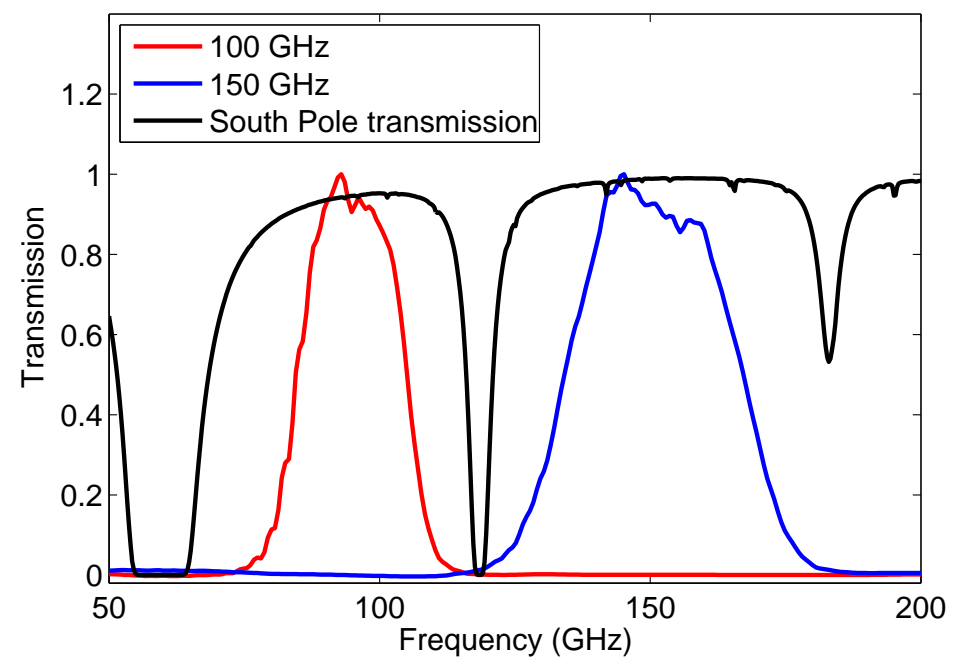

Figure 4. Keck Array averaged frequency response spectrum for $100 \mathrm{GHz}$ (red) and $150 \mathrm{GHz}$ (blue) detectors, with the atmospheric transmission at the South Pole for comparison. The detector responses are normalized to unit peak and have had the source spectrum $\left(\sim \nu^{2}\right)$ divided out. The atmospheric transmission spectrum is in units of fractional power transmitted.

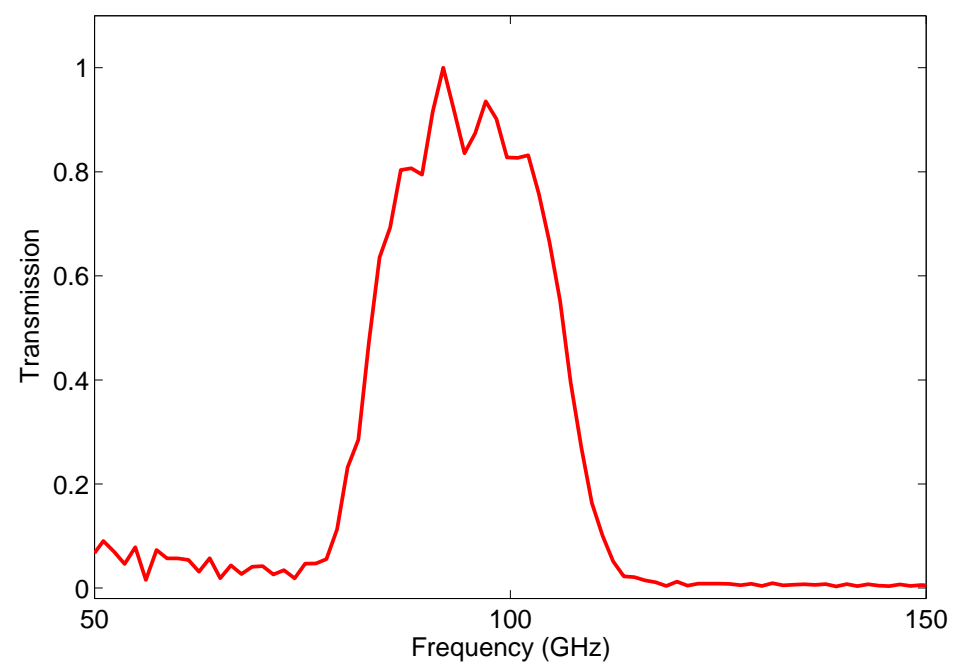

Figure 5. Preliminary spectrum of a single BICEP3 detector, normalized to unit peak and with the source spectrum $\left(\sim \nu^{2}\right)$ divided out.

To produce spectra, the interferograms are low-pass filtered, aligned on the white-light fringe (zero path length difference), and windowed before being Fourier transformed to obtain the frequency response $S(\nu)$. Figure 4 shows array-averaged spectra of the Keck Array (2 receivers at $100 \mathrm{GHz}$ and 3 receivers at $150 \mathrm{GHz}$ ), while Figure 5 shows an example spectrum of a single BICEP3 detector. As designed, the spectra avoid the atmospheric oxygen lines at 118 and $183 \mathrm{GHz}$.

\subsection{Bandwidths and Band Centers}

For each detector's best spectrum (i.e. largest amplitude raw interferogram), we compute the band center, defined as

$$
\langle\nu\rangle=\int \nu S(\nu) d \nu,
$$


and the bandwidth, defined as

$$
\Delta \nu=\frac{\left(\int S(\nu) d \nu\right)^{2}}{\int S^{2}(\nu) d \nu} .
$$

Figures 6 and 7 show histograms of the computed band centers and bandwidths for each of the 2014 Keck Array receivers. Much of the variation in each focal plane is driven by tile-to-tile differences, as illustrated by the high band centers in Receiver 1 (Figure 6). Average values for each of the 20 Keck Array tiles are shown in Table 1. Averaged over the entire array, the $100 \mathrm{GHz}$ band center and bandwidth are $94.8 \pm 0.8 \mathrm{GHz}$ and $25.5 \pm 0.4 \mathrm{GHz}$, while for $150 \mathrm{GHz}$ they are $150.7 \pm 1.9 \mathrm{GHz}$ and $41.8 \pm 1.4 \mathrm{GHz}$. The uncertainties indicate the scatter across all detectors in each band.
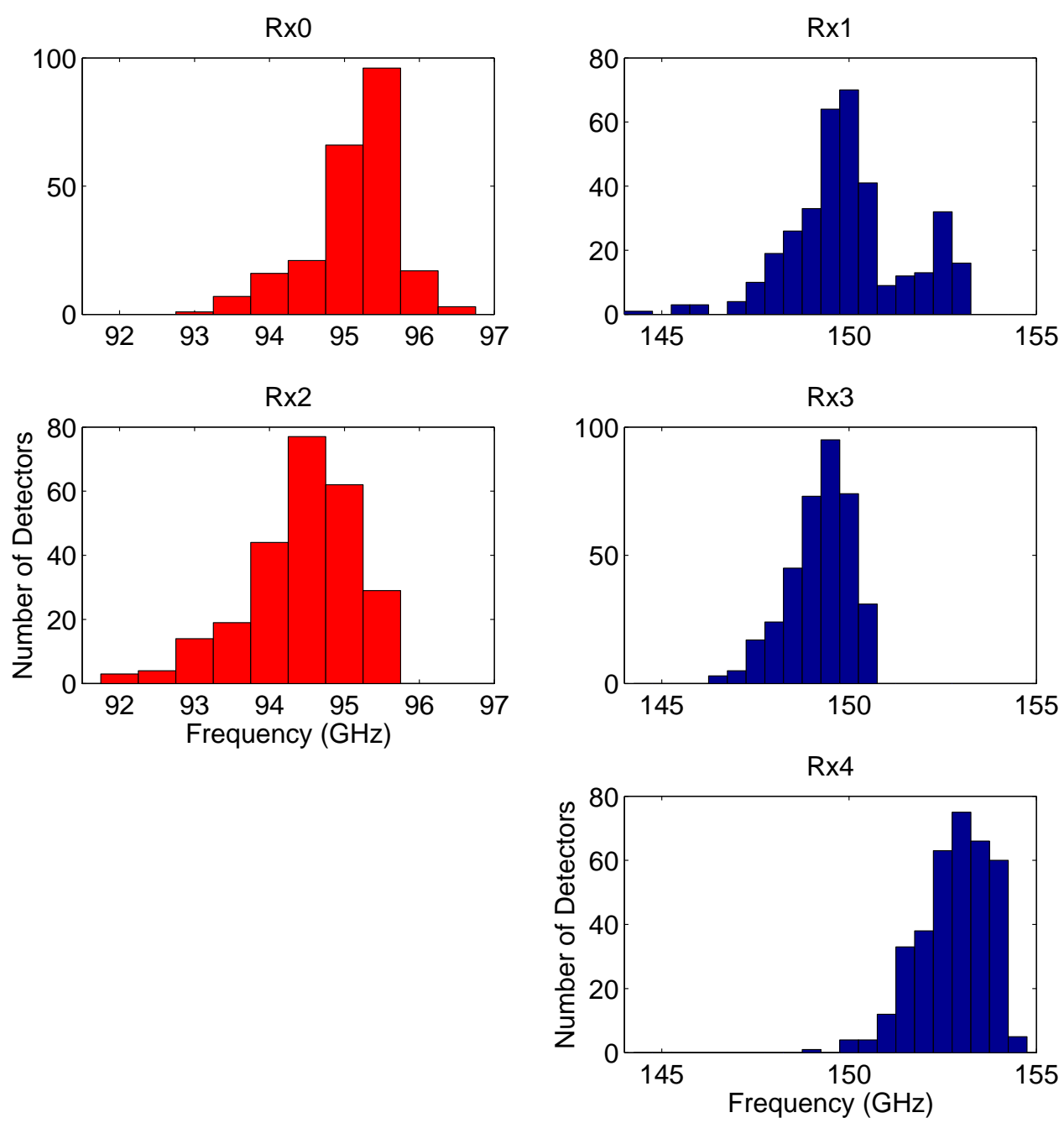

Figure 6. Histograms of band centers for each of the 2014 Keck Array receivers, in $0.5 \mathrm{GHz}$ bins. Rx0 and Rx2 are centered at $95 \mathrm{GHz}$ while $\mathrm{Rx} 1, \mathrm{Rx} 3$, and $\mathrm{Rx} 4$ are at $150 \mathrm{GHz}$.

\section{ATMOSPHERIC MODELING}

The am software is a free, open source program that rapidly provides a model for the radiative characteristics of the atmosphere across an observing band. Using am to model the South Pole atmosphere, we find that the variation in the predicted absolute calibration among detectors is consistent with the measured variation in 

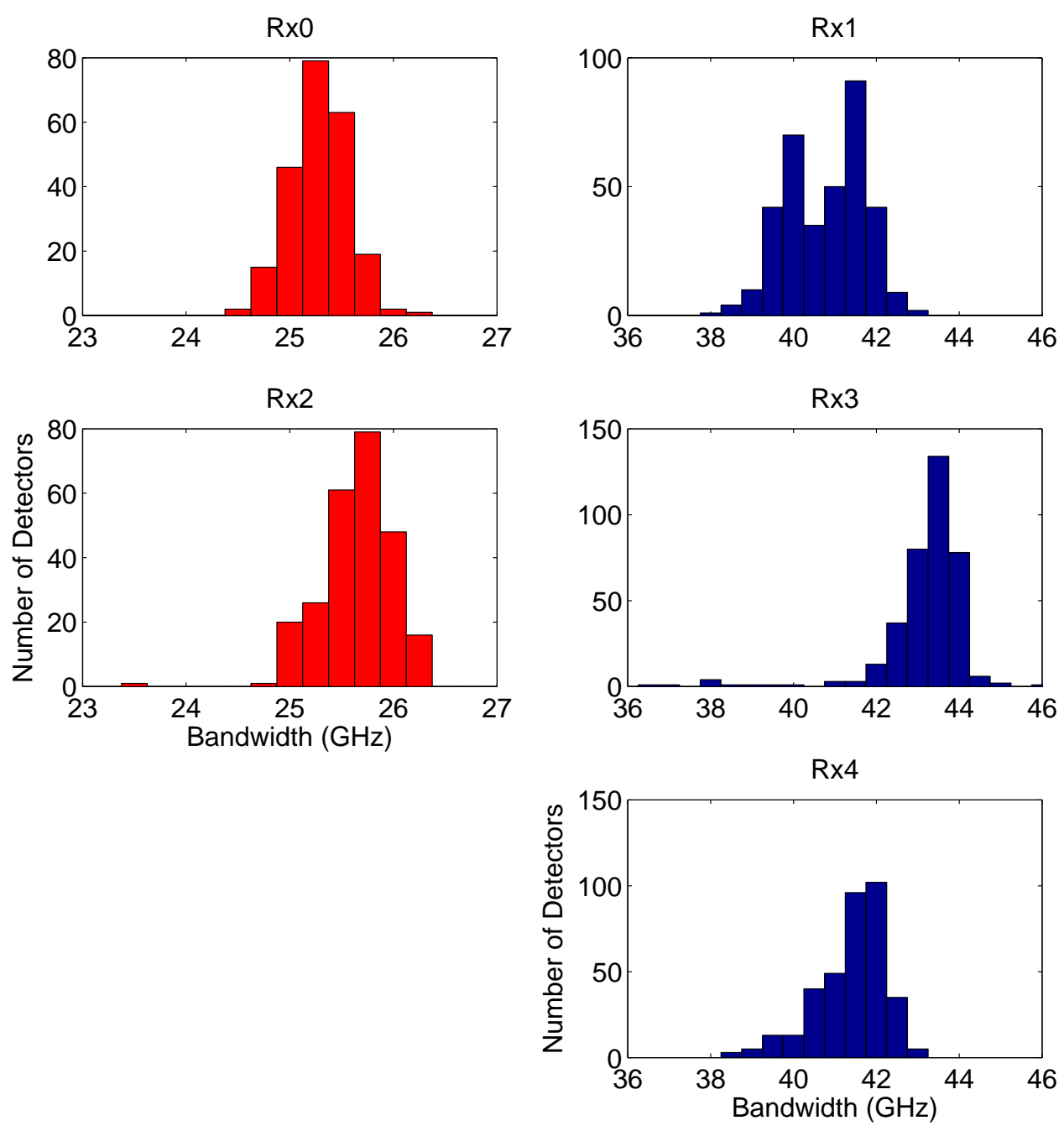

Figure 7. Histograms of bandwidths for each of the 2014 Keck Array receivers. Rx0 and Rx2 are centered at $95 \mathrm{GHz}$ while $\mathrm{Rx} 1, \mathrm{Rx} 3$, and $\mathrm{Rx} 4$ are at $150 \mathrm{GHz}$.

detector bandpasses. Figure 8 compares predicted zenith temperatures (obtained with measured spectra and $a m$ ) to measured absolute calibrations for every detector on a single Keck Array receiver. They correlate as expected, providing a useful cross check on our calibration procedure. These atmospheric models also predict steadier conditions at $100 \mathrm{GHz}$ compared to $150 \mathrm{GHz}$ because of the reduced influence of water vapor. Preliminary Keck Array data with simultaneous observations at 100 and $150 \mathrm{GHz}$ support this.

\section{CONCLUSIONS}

Knowledge of the detailed spectral response of our detectors is important for determining the degree of potential coupling to atmospheric or astrophysical line emission, in addition to providing cross checks on potential systematic effects in polarization data such as bandpass or relative gain mismatch between orthogonally polarized, co-located detectors.

In these proceedings, we have characterized the spectral response of the every Keck Array detector in its 2014 observing configuration. For our $100 \mathrm{GHz}$ detectors, we have measured band centers of $94.8 \pm 0.8 \mathrm{GHz}$, and for the $150 \mathrm{GHz}$ detectors $150.7 \pm 1.9 \mathrm{GHz}$. We have also presented preliminary spectra of existing BICEP3 


\begin{tabular}{|c|c|c|c|}
\hline \multicolumn{2}{|c|}{ Keck Array 2014 } & \multicolumn{2}{|c|}{ Spectral Parameter } \\
\cline { 3 - 4 } & Tile 1 & Band Center, $\langle\nu\rangle(\mathrm{GHz})$ & Bandwidth, $\Delta \nu(\mathrm{GHz})$ \\
\hline \multirow{5}{*}{ Receiver 0 } & Tile 2 & $95.53 \pm 0.45$ & $25.28 \pm 0.27$ \\
& Tile 3 & $94.86 \pm 0.61$ & $25.33 \pm 0.20$ \\
& Tile 4 & $94.92 \pm 0.59$ & $25.09 \pm 0.27$ \\
& All tiles & $95.13 \pm 0.60$ & $25.45 \pm 0.22$ \\
\hline \multirow{5}{*}{ Receiver 1 1} & Tile 1 & $152.13 \pm 0.76$ & $25.28 \pm 0.27$ \\
& Tile 2 & $149.62 \pm 0.79$ & $41.72 \pm 0.45$ \\
& Tile 3 & $149.16 \pm 1.09$ & $41.14 \pm 0.52$ \\
& Tile 4 & $149.14 \pm 1.28$ & $39.67 \pm 0.47$ \\
& All tiles & $149.97 \pm 1.58$ & $40.78 \pm 0.95$ \\
\hline \multirow{5}{*}{ Receiver 2 2} & Tile 1 & $94.32 \pm 0.60$ & $25.64 \pm 0.22$ \\
& Tile 2 & $94.71 \pm 0.80$ & $25.70 \pm 0.36$ \\
& Tile 3 & $94.77 \pm 0.54$ & $25.28 \pm 0.25$ \\
& Tile 4 & $93.97 \pm 0.70$ & $25.93 \pm 0.21$ \\
& All tiles & $94.43 \pm 0.73$ & $25.64 \pm 0.35$ \\
\hline & Tile 1 & $148.75 \pm 0.80$ & $43.39 \pm 0.96$ \\
& Tile 2 & $149.79 \pm 0.61$ & $43.47 \pm 0.41$ \\
Receiver 3 3 & Tile 3 & $149.20 \pm 0.82$ & $42.90 \pm 1.18$ \\
& Tile 4 & $149.20 \pm 0.78$ & $43.00 \pm 1.34$ \\
& All tiles & $149.22 \pm 0.85$ & $43.19 \pm 1.05$ \\
\hline & Tile 1 & $152.81 \pm 1.01$ & $41.56 \pm 0.72$ \\
& Tile 2 & $153.11 \pm 1.08$ & $40.59 \pm 0.97$ \\
& Tile 3 & $152.59 \pm 0.55$ & $41.97 \pm 0.33$ \\
& Tile 4 & $152.76 \pm 0.93$ & $41.49 \pm 0.53$ \\
& All tiles & $152.82 \pm 0.94$ & $41.40 \pm 0.84$ \\
\hline
\end{tabular}

Table 1. Measured spectral parameters for Keck Array receivers in 2014. Shown are the mean value and the detector-todetector scatter.

detectors. We have shown that applying our bandpasses to simulated South Pole skies returns expected zenith temperatures consistent with our measured per-detector absolute calibrations.

To take these spectra, we have constructed a field-deployable Fourier Transform Spectrometer which is capable of characterizing the entire Keck Array in several days, and we will do the same for BICEP3 when it deploys to the South Pole in 2014-2015. As new experiments come online in the coming years, it will become crucial to efficiently calibrate large numbers of detectors on site. Our measurements of the 5000+ detectors in the Keck Array and BICEP3 represent a first step towards this goal.

\section{ACKNOWLEDGMENTS}

The Keck Array and BICEP3 projects have been made possible through support from the National Science Foundation (grant Nos. 0742818, 0742592, 1044978, 1110087, 1145172, 1313158, 1313010, 1313062, 1313287, 1056465, and 0960243), the W. M. Keck Foundation, the Canada Foundation for Innovation, and the British Columbia Development Fund. The development of antenna-coupled detector technology was supported by the JPL Research and Technology Development Fund and grants 06-ARPA206-0040 and 10-SAT10-0017 from the NASA ARPA and SAT programs. The development and testing of focal planes were supported by the Gordon and Betty Moore Foundation at Caltech. The computations in these proceedings were run on the Odyssey cluster supported by the FAS Science Division Research Computing Group at Harvard University. Tireless administrative support was provided by Irene Coyle, Kathy Deniston, Donna Hernandez, and Dana Volponi.

We are grateful to Robert Schwarz as our 2011-2014 Keck Array winterover. We thank the staff of the US Antarctic Program and in particular the South Pole Station without whose help this research would not have been possible. We thank our BICEP1, BICEP2, and SPIDER colleagues for useful discussions and shared expertise. 


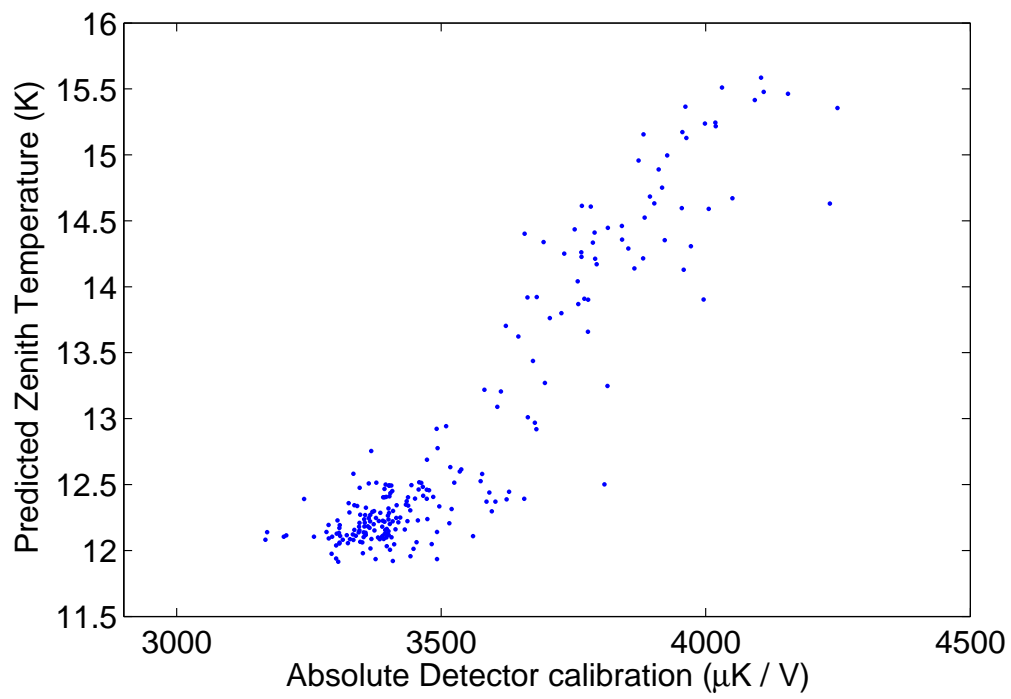

Figure 8. Per-detector absolute calibrations (derived from correlation with CMB) compared with predicted zenith temperatures (derived from measured spectra and predicted South Pole skies) for a single $150 \mathrm{GHz}$ Keck Array receiver. Each point represents a separate detector on the focal plane. Differences in measured absolute calibrations are consistent with the predicted zenith temperatures, driven by differences in measured bandpasses.

\section{REFERENCES}

[1] BICEP2 Collaboration, Ade, P. A. R., Aikin, R. W., Barkats, D., Benton, S. J., Bischoff, C. A., Bock, J. J., Brevik, J. A., Buder, I., Bullock, E., Dowell, C. D., Duband, L., Filippini, J. P., Fliescher, S., Golwala, S. R., Halpern, M., Hasselfield, M., Hildebrandt, S. R., Hilton, G. C., Hristov, V. V., Irwin, K. D., Karkare, K. S., Kaufman, J. P., Keating, B. G., Kernasovskiy, S. A., Kovac, J. M., Kuo, C. L., Leitch, E. M., Lueker, M., Mason, P., Netterfield, C. B., Nguyen, H. T., O'Brient, R., Ogburn, R. W., Orlando, A., Pryke, C., Reintsema, C. D., Richter, S., Schwarz, R., Sheehy, C. D., Staniszewski, Z. K., Sudiwala, R. V., Teply, G. P., Tolan, J. E., Turner, A. D., Vieregg, A. G., Wong, C. L., and Yoon, K. W., "Detection of B-Mode Polarization at Degree Angular Scales by BICEP2," Physical Review Letters 112, 241101 (June 2014).

[2] Sheehy, C. D., Ade, P. A. R., Aikin, R. W., Amiri, M., Benton, S., Bischoff, C., Bock, J. J., Bonetti, J. A., Brevik, J. A., Burger, B., Dowell, C. D., Duband, L., Filippini, J. P., Golwala, S. R., Halpern, M., Hasselfield, M., Hilton, G., Hristov, V. V., Irwin, K., Kaufman, J. P., Keating, B. G., Kovac, J. M., Kuo, C. L., Lange, A. E., Leitch, E. M., Lueker, M., Netterfield, C. B., Nguyen, H. T., Ogburn, IV, R. W., Orlando, A., Pryke, C. L., Reintsema, C., Richter, S., Ruhl, J. E., Runyan, M. C., Staniszewski, Z., Stokes, S., Sudiwala, R., Teply, G., Thompson, K. L., Tolan, J. E., Turner, A. D., Wilson, P., and Wong, C. L., "The Keck Array: a pulse tube cooled CMB polarimeter," in [Society of Photo-Optical Instrumentation Engineers (SPIE) Conference Series], Society of Photo-Optical Instrumentation Engineers (SPIE) Conference Series 7741 (July 2010).

[3] Ogburn, R. W., Ade, P. A. R., Aikin, R. W., Amiri, M., Benton, S. J., Bischoff, C. A., Bock, J. J., Bonetti, J. A., Brevik, J. A., Bullock, E., Burger, B., Davis, G., Dowell, C. D., Duband, L., Filippini, J. P., Fliescher, S., Golwala, S. R., Gordon, M., Halpern, M., Hasselfield, M., Hilton, G., Hristov, V. V., Hui, H., Irwin, K., Kaufman, J. P., Keating, B. G., Kernasovskiy, S. A., Kovac, J. M., Kuo, C. L., Leitch, E. M., Lueker, M., Montroy, T., Netterfield, C. B., Nguyen, H. T., O’Brient, R., Orlando, A., Pryke, C. L., Reintsema, C., Richter, S., Ruhl, J. E., Runyan, M. C., Schwarz, R., Sheehy, C. D., Staniszewski, Z. K., Sudiwala, R. V., Teply, G. P., Thompson, K., Tolan, J. E., Turner, A. D., Vieregg, A. G., Wiebe, D. V., Wilson, P., and Wong, C. L., "BICEP2 and Keck Array operational overview and status of observations," in [Society of Photo-Optical Instrumentation Engineers (SPIE) Conference Series], Society of Photo-Optical Instrumentation Engineers (SPIE) Conference Series 8452 (Sept. 2012). 
[4] Ahmed, Z. et al., "BICEP3: A Next-Generation Refractor for Inflationary CMB Polarization," in [These proceedings], Society of Photo-Optical Instrumentation Engineers (SPIE) Conference Series 9153 (2014).

[5] Planck Collaboration, Ade, P. A. R., Aghanim, N., Alves, M. I. R., Armitage-Caplan, C., Arnaud, M., Ashdown, M., Atrio-Barandela, F., Aumont, J., Baccigaluppi, C., and et al., "Planck 2013 results. XIII. Galactic CO emission," ArXiv e-prints (Mar. 2013).

[6] Vieregg, A. G., Ade, P. A. R., Aikin, R., Bischoff, C., Bock, J. J., Bonetti, J. A., Bradford, K. J., Brevik, J. A., Dowell, C. D., Duband, L., Filippini, J. P., Fliescher, S., Golwala, S. R., Gordon, M. S., Halpern, M., Hilton, G., Hristov, V. V., Irwin, K., Kernasovskiy, S., Kovac, J. M., Kuo, C. L., Leitch, E., Lueker, M., Montroy, T., Netterfield, C. B., Nguyen, H. T., O’Brient, R., Ogburn, R. W., Pryke, C., Ruhl, J. E., Runyan, M., Schwarz, R., Sheehy, C., Staniszewski, Z., Sudiwala, R., Teply, G., Tolan, J., Turner, A. D., Wilson, P., and Wong, C. L., "Optical characterization of the Keck array polarimeter at the South Pole," in [Society of Photo-Optical Instrumentation Engineers (SPIE) Conference Series], Society of Photo-Optical Instrumentation Engineers (SPIE) Conference Series 8452 (Sept. 2012).

[7] Buder, I. et al., "BICEP2 and Keck Array: Upgrades and Improved Beam Characterization," in [These proceedings], Society of Photo-Optical Instrumentation Engineers (SPIE) Conference Series 9153 (2014).

[8] BICEP2 and Keck Array Collaborations, "Optical Characterization and Performance of the BICEP2 and Keck Array Experiments," In prep. (2014).

[9] BICEP2 Collaboration, "BICEP2 III: Instrumental Systematics," In prep. (2014).

[10] BICEP2 Collaboration, Ade, P. A. R., Aikin, R. W., Amiri, M., Barkats, D., Benton, S. J., Bischoff, C. A., Bock, J. J., Brevik, J. A., Buder, I., Bullock, E., Davis, G., Dowell, C. D., Duband, L., Filippini, J. P., Fliescher, S., Golwala, S. R., Halpern, M., Hasselfield, M., Hildebrandt, S. R., Hilton, G. C., Hristov, V. V., Irwin, K. D., Karkare, K. S., Kaufman, J. P., Keating, B. G., Kernasovskiy, S. A., Kovac, J. M., Kuo, C. L., Leitch, E. M., Llombart, N., Lueker, M., Netterfield, C. B., Nguyen, H. T., O'Brient, R., Ogburn, IV, R. W., Orlando, A., Pryke, C., Reintsema, C. D., Richter, S., Schwarz, R., Sheehy, C. D., Staniszewski, Z. K., Story, K. T., Sudiwala, R. V., Teply, G. P., Tolan, J. E., Turner, A. D., Vieregg, A. G., Wilson, P., Wong, C. L., and Yoon, K. W., "BICEP2 II: Experiment and Three-Year Data Set," ArXiv e-prints: 1403.4302 (Mar. 2014).

[11] BICEP2 and Keck Array Collaborations, "Antenna-Array-Coupled TES Bolometers used in BICEP2, Keck Array, and SPIDER," In prep. (2014).

[12] Kuo, C. L., Bock, J. J., Bonetti, J. A., Brevik, J., Chattopadhyay, G., Day, P. K., Golwala, S., Kenyon, M., Lange, A. E., LeDuc, H. G., Nguyen, H., Ogburn, R. W., Orlando, A., Transgrud, A., Turner, A., Wang, G., and Zmuidzinas, J., "Antenna-coupled TES bolometer arrays for CMB polarimetry," in [Society of PhotoOptical Instrumentation Engineers (SPIE) Conference Series], Society of Photo-Optical Instrumentation Engineers (SPIE) Conference Series 7020 (Aug. 2008).

[13] Ade, P. A. R., Pisano, G., Tucker, C., and Weaver, S., "A review of metal mesh filters," in [Society of PhotoOptical Instrumentation Engineers (SPIE) Conference Series], Society of Photo-Optical Instrumentation Engineers (SPIE) Conference Series 6275 (July 2006).

[14] Lesurf, J., [Millimetre-Wave Optics, Devices and Systems], Taylor \& Francis (1990).

[15] Martin, D. H. and Puplett, E., "Polarised interferometric spectrometry for the millimeter and submillimeter spectrum.," Infrared Physics 10, 105-109 (1970).

[16] Kraus, J., [Radio astronomy], McGraw-Hill (1986). 